\title{
Drawing Hamiltonian cycles with no large angles*
}

\author{
Adrian Dumitrescu ${ }^{\dagger}$ \\ Department of Computer Science \\ University of Wisconsin-Milwaukee \\ Wisconsin, USA \\ dumitres@uwm.edu
}

\author{
János Pach ${ }^{\ddagger}$ \\ Department of Mathematics \\ Ecole Polytechnique Fédérale de Lausanne \\ and City College, New York \\ pach@cims.nyu.edu
}

\author{
Géza Tóth ${ }^{\S}$ \\ Alfred Rényi Institute of Mathematics \\ Budapest, Hungary \\ geza@renyi.hu
}

\begin{abstract}
Submitted: Sep 17, 2010; Accepted: May 9, 2012; Published: Jun 6, 2012
Mathematics Subject Classification: 52C35
\end{abstract}

\begin{abstract}
Let $n \geqslant 4$ be even. It is shown that every set $S$ of $n$ points in the plane can be connected by a (possibly self-intersecting) spanning tour (Hamiltonian cycle) consisting of $n$ straight-line edges such that the angle between any two consecutive edges is at most $2 \pi / 3$. For $n=4$ and 6 , this statement is tight. It is also shown that every even-element point set $S$ can be partitioned into at most two subsets, $S_{1}$ and $S_{2}$, each admitting a spanning tour with no angle larger than $\pi / 2$. Fekete and Woeginger conjectured that for sufficiently large even $n$, every $n$-element set admits such a spanning tour. We confirm this conjecture for point sets in convex position. A much stronger result holds for large point sets randomly and uniformly selected from an open region bounded by finitely many rectifiable curves: for any $\varepsilon>0$, these sets almost surely admit a spanning tour with no angle larger than $\varepsilon$.
\end{abstract}

Keywords: Hamiltonian cycle, turning angle, geometric graph.

*An extended abstract of this paper appeared in the Proceedings of the 17th International Symposium on Graph Drawing, Chicago, Sept. 2009, Volume 5849 of LNCS, Springer Verlag, March 2010, pp. 3-14.

†Supported in part by NSF grants CCF-0444188 and DMS-1001667, and by the Discrete and Convex Geometry project, in the framework of the European Community's "Structuring the European Research Area" program. Part of the research by this author was done at Alfred Rényi Institute of Mathematics in Budapest, and at Ecole Polytechnique Fédérale de Lausanne.

${ }^{\ddagger}$ Research partially supported by NSF grant CCF-08-30272, grants from OTKA, SNF, and PSCCUNY.

${ }^{\S}$ Supported by OTKA K 83767 and NN 102029, and by TÁMOP - 4.2.2.B-10/1-2010-0009, in the framework of the project "Talent care and cultivation in the scientific workshops of BME". 


\section{Introduction}

In the Euclidean traveling salesman problem (TSP), given a set of points in the plane, one seeks a shortest tour that visits each point. In recent years, there has been an increased interest in studying tours that optimize objective functions related to angles between consecutive edges in the tour, rather than the length. The problem has applications in motion planning, where restrictions on turning angles have to be enforced. For example, an aircraft or a boat moving at high speed, required to pass through a set of given locations, cannot make sharp turns in its motion. This and other applications to planning curvature-constrained paths for auto-vehicles and aircraft are discussed in $[2,7,13,14]$.

Consider a set of $n \geqslant 2$ points. A spanning tour is a directed Hamiltonian cycle, drawn with straight-line edges; if $n=2$ the tour consists of the two edges, with opposite orientations, connecting the two points. When three points, $p_{1}, p_{2}$, and $p_{3}$, are traversed in this order, their rotation angle $\angle p_{1} p_{2} p_{3}$ is the angle in $[0, \pi]$ determined by segments $p_{1} p_{2}$ and $p_{2} p_{3}$; see Figure 1. If $p_{3}$ is on the left (resp. right) side of the oriented line $\overrightarrow{p_{1} p_{2}}$ we say that the tour, or path makes a left (resp. right) turn at $p_{2}$. If a tour (or path) makes only right turns, we call it pseudo-convex. If all of its rotation angles are at most $\pi / 2$, we call it an acute tour (or path). If all rotation angles are at least $\pi / 2$, the tour (or path) is obtuse.

Given a set $A$ of angles, the angle-restricted tour (ART) problem is to decide whether a set $S$ of $n$ points in the plane allows a (possibly self-intersecting) spanning tour such that all the $n$ angles between consecutive segments belong to the set $A$; see [12].

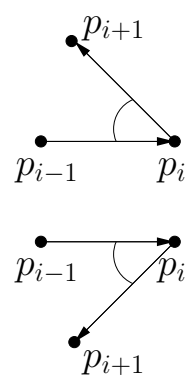

(a)
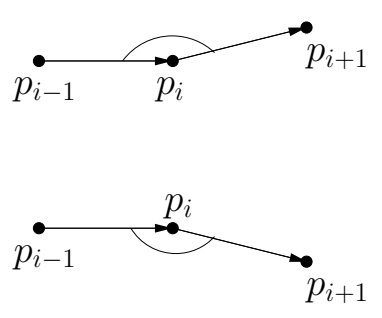

(b)

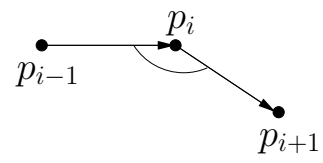

(c)

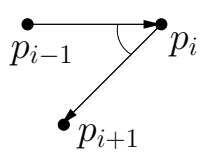

(d)

Figure 1: (a) acute (b) obtuse (c) obtuse and pseudo-convex (d) acute and pseudo-convex

Fekete and Woeginger [12] proved that every finite set of at least five points admits a pseudo-convex tour and a non-intersecting pseudo-convex spanning path. They also noticed that every $n$-element point set $S$ admits an acute spanning path. To see this, start at any point $p_{1} \in S$. Assuming that the initial portion $p_{1} \ldots p_{i}$ of such a path has already been defined and $i<n$, let $p_{i+1}$ be an element of $S \backslash\left\{p_{1}, \ldots, p_{i}\right\}$ farthest away from $p_{i}$. It is easy to check that the resulting path $p_{1} \ldots p_{n}$ is acute. It is also clear that such a path cannot be always completed to an acute tour. Indeed, if all points are on a line and $n$ is odd, then along any (spanning) tour, one of the rotation angles must be equal to $\pi$. 
The question arises: Does every even-element point set admit a tour with small rotation angles? More precisely, given an $n$-element point set $S$ in the plane, where $n$ is even, let $\alpha=\alpha(S) \geqslant 0$ denote the smallest angle such that $S$ admits a (spanning) tour with the property that all of its rotation angles belong to $[0, \alpha]$. Finally, let $\alpha(n)$ be the maximum of $\alpha(S)$ over all $n$-element point sets in the plane. Trivially, $\alpha(2)=0$. The 4 -element point set formed by the 3 vertices and the center of an equilateral triangle shows that $\alpha(4) \geqslant 2 \pi / 3$. The 6 -point configuration depicted in Fig. 2 (left) shows that $\alpha(6) \geqslant 2 \pi / 3$.
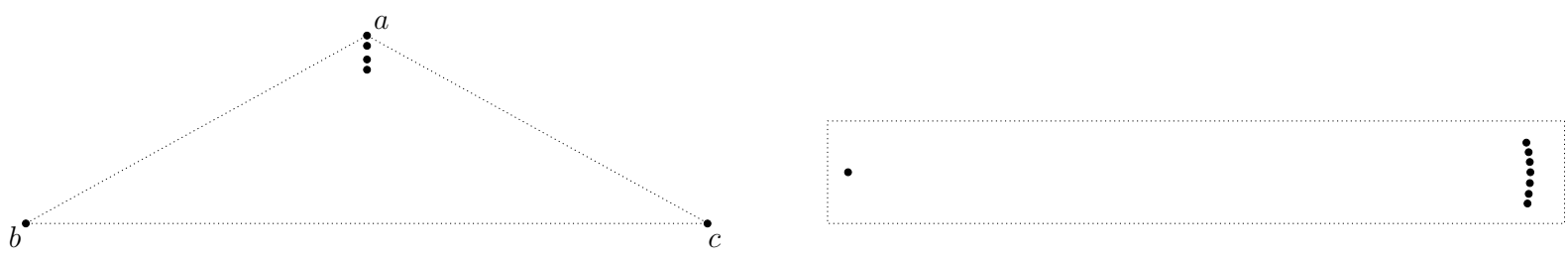

Figure 2: Left: $\triangle a b c$ is an isosceles triangle with $\angle b a c=2 \pi / 3$. Point $a$ and the 3 points below it are placed on the altitude of the triangle, and very closely inter-spaced. Every tour on these 6 points has a rotation angle of at least $2 \pi / 3-\varepsilon$. Right: $n-1$ equidistant points very closely inter-spaced on a small circular arc of a circle, and one point at the center. Every tour on these $n$ points has a rotation angle of at least $\pi / 2-\varepsilon$.

In this note we show that $\alpha(n) \leqslant 2 \pi / 3$, for all even $n \geqslant 4$.

Theorem 1. Let $n \geqslant 4$ be even. Every set of $n$ points in the plane admits a spanning tour such that all of its rotation angles are at most $2 \pi / 3$. This bound is tight for $n=4,6$. Such a tour can be computed in $O\left(n^{4 / 3} \log ^{1+\varepsilon} n\right)$ time, for every $\varepsilon>0$.

It remains open whether the bound $2 \pi / 3$ can be replaced by $\pi / 2$, for every even $n \geqslant 8$, as was conjectured in [12]. In other words, every $n$-element set may admit an acute tour, whenever $n \geqslant 8$ is even. The point set depicted in Fig. 2 (right) demonstrates that this statement, if true, cannot be improved. That is, we have $\alpha(n) \geqslant \pi / 2$, for all even $n \geqslant 8$.

We confirm three weaker versions of this statement. In Section 4 , we show that if we enforce acute rotation angles, two tours instead of one will certainly suffice.

Theorem 2. Let $n \geqslant 8$ be even.

(i) Every set of $n$ points in the plane can be partitioned into two even parts, each of which admits an acute spanning tour. Given the $n$ points, the two tours can be computed in $O(n)$ time.

(ii) Every set of $n$ points in the plane can be partitioned into two parts of sizes $2\left\lfloor\frac{n}{4}\right\rfloor$ and $2\left\lceil\frac{n}{4}\right\rceil$, each of which admits an acute spanning tour. Given the $n$ points, the two tours can be computed in $O\left(n^{4 / 3} \log ^{1+\varepsilon} n\right)$ time, for every $\varepsilon>0$.

In Section 5, we prove the existence of an acute tour in the special case when the points are in convex position.

Theorem 3. Every even set $S$ of $n$ points in the plane in convex position, with $n \geqslant 12$, admits an acute spanning tour. Given the $n$ points, such a tour can be computed in $O(n)$ time. 
A much stronger statement holds for random point sets, uniformly selected from a not necessarily connected region.

Theorem 4. Let $B$ be an open region in the plane bounded by finitely many rectifiable Jordan curves and let $S$ be a set of $n$ points, randomly and uniformly selected from $B$. Then, for any $\varepsilon>0$, the point set $S$ almost surely admits a spanning tour with no rotation angle larger than $\varepsilon$, as $n$ tends to infinity.

The last result easily generalizes to higher dimensions.

Related problems and results. Various angle conditions imposed on geometric graphs (graphs with straight-line edges) drawn on a given set of points have been studied in [3, $4,5,6,12]$. In particular, Fekete and Woeginger [12] have focused on rotation angles of Hamiltonian cycles and paths and raised many challenging questions. For instance, they conjectured that any point set admits a (possibly self-intersecting) Hamiltonian path in which each turning angle is at most $5 \pi / 6$. Bárány, Pór, and Valtr [6] obtained a first partial result by showing that any point set admits a (possibly self-intersecting) Hamiltonian path in which each turning angle is at most $8 \pi / 9$.

Aichholzer et al. [3] studied similar questions for planar geometric graphs. Among other results, they showed that any point set in general position in the plane admits a non-intersecting Hamiltonian (spanning) path with the property that each rotation angle is at most $3 \pi / 4$. They also conjectured that this value can be replaced by $\pi / 2$. Arkin et al. introduced the notion of reflexivity of a point set, as the minimum number of reflex vertices in a polygonalization (i.e., simple polygon) of the set [5]. They gave estimates for the maximum reflexivity of an $n$-element point set. Recently, Ackerman et al. have made further progress on this problem [1].

\section{Balanced partitions}

It is well known (see, e.g. [10], Section 6.6) that every region (every continuous probability measure) in the plane can be cut into four parts of equal area (measure) by two orthogonal lines. This statement immediately implies:

Lemma 1. Given a set $S$ of $n \geqslant 8$ points in the plane ( $n$ even), one can always find two orthogonal lines $\ell_{1}, \ell_{2}$ and a partition $S=S_{1} \cup S_{2} \cup S_{3} \cup S_{4}$ with $\left|S_{1}\right|=\left|S_{3}\right|=\left\lfloor\frac{n}{4}\right\rfloor$, $\left|S_{2}\right|=\left|S_{4}\right|=\left\lceil\frac{n}{4}\right\rceil$ such that $S_{1}$ and $S_{3}$ belong to two opposite closed quadrants determined by $\ell_{1}$ and $\ell_{2}$, and $S_{2}$ and $S_{4}$ belong to the other two opposite quadrants.

Proof. By a standard compactness argument, it is sufficient to prove this statement for point sets $S$ in general position, in the sense that no 3 points of $S$ are on a line, no 3 determine a right angle, and no two segments spanned by 4 points are orthogonal to each other. Choose a very small $\varepsilon>0$ and replace each point $p \in S$ by a disk of radius $\varepsilon$ around $p$. Applying the above mentioned result from [10] to the union of these $n$ disks, we obtain two orthogonal lines that meet the requirements of the lemma. 
Lemma 2. Given a set $S$ of $n$ points in the plane ( $n$ even), there exist three concurrent lines such that the angle between any two of them is $\pi / 3$, and there is a partition $S=$ $S_{1} \cup \ldots \cup S_{6}$ with $\left|S_{1}\right|=\left|S_{4}\right|,\left|S_{2}\right|=\left|S_{5}\right|$, and $\left|S_{3}\right|=\left|S_{6}\right|$, such that $S_{i}$ is contained in the ith closed angular region (wedge) determined by the lines, in counterclockwise order.

Proof. Just like before, by compactness, it is sufficient to prove the statement for point sets in general position. This time, it is convenient to assume that no 3 points of $S$ determine an angle which is an integer multiple of $\pi / 3$, and there are no 2 pairs of points such that the angle between their connecting lines is an integer multiple of $\pi / 3$.

Choose again a very small $\varepsilon>0$ and replace each point $p \in S$ by a disk $D_{p}$ of radius $\varepsilon$ centered at $p$. Approximate very closely the union of these disks by a continuous measure $\mu$ which is strictly positive on every Jordan region in the plane and for which $\mu\left(\mathbb{R}^{2}\right)=n$ and $\left|\mu\left(D_{p}\right)-1\right|<\varepsilon$ for every $p \in S$.

We say that a line $\ell$ is a bisecting line with respect to the continuous measure $\mu$ if the measures of both half-planes bounded by $\ell$ are equal to $n / 2$. Clearly, there is a unique bisecting line parallel to every direction, and this line changes continuously as the direction varies. Choose three bisecting lines $\ell_{1}, \ell_{2}, \ell_{3}$ such that the angle between any two of them is $\pi / 3$. By changing the direction of $\ell_{1}$, we can achieve that these lines pass through the same point. Indeed, as we turn $\ell_{1}$ by $\pi / 3$, the crossing point of the other two lines moves from one side of $\ell_{1}$ to the other. Therefore, there is an intermediate position in which the three lines pass through the same point.

An easy case analysis shows that if $\varepsilon$ was sufficiently small, then either no $\ell_{i}$ intersects any disk $D_{p}$ or there is one $\ell_{i}$ that intersects two $D_{p}$ 's and the others do not intersect any. In the former case, the lines satisfy the conditions in the lemma, in the latter one, they can be slightly perturbed so as to meet the requirements.

Given a set $S$ of $n$ points in general position in the plane (i.e., no three points are collinear), a line passing through two elements of $S$ is called a halving line if there are $\lfloor(n-2) / 2\rfloor$ points on one of its sides and $\lceil(n-2) / 2\rceil$ points on the other [15]. The number of halving lines of an $n$-element point set in the plane is bounded from above by $O\left(n^{4 / 3}\right)$, as was established by Dey [11]. It is also known that the set of halving lines can be computed in $O\left(n^{4 / 3} \log ^{1+\varepsilon} n\right)$ time [8], for every $\varepsilon>0$.

Remark. Starting with an arbitrary halving line $\ell$ and following the rotation scheme described in [15], one can enumerate all halving lines for $S$. Using this approach, one obtains algorithmic proofs of Lemmas 1 and 2 that run in $O\left(n^{4 / 3} \log ^{1+\varepsilon} n\right)$ time, for every $\varepsilon>0$.

\section{Constructing a tour with rotation angles at most $2 \pi / 3$}

In this section, we prove Theorem 1. As we mentioned in the Introduction, for small even values of $n$, namely for $n=4$ and $n=6$, we need to allow rotation angles as large as $2 \pi / 3$. Here we show that this value suffices for all even $n$. 
Let $\ell_{1}, \ell_{2}, \ell_{3}$ be three concurrent lines satisfying the conditions of Lemma 2. They divide the plane into six wedges.

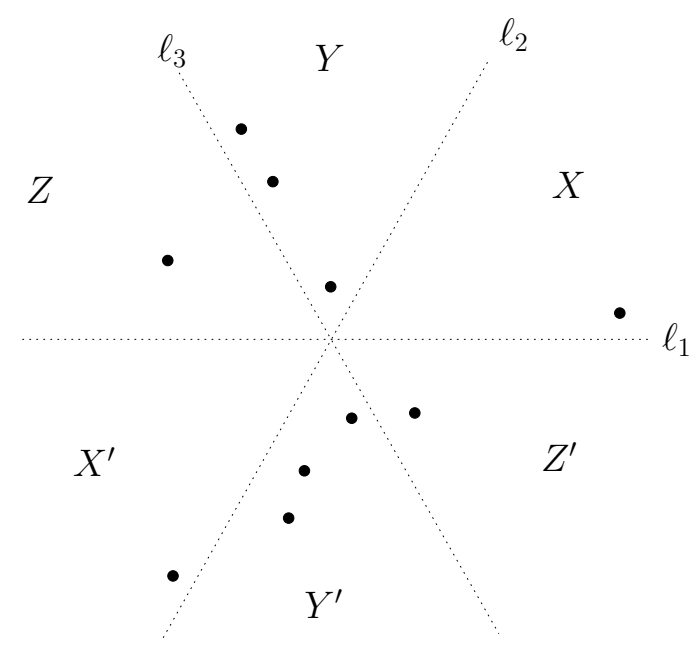

Figure 3: Three concurrent bisecting lines of $S: \ell_{1}, \ell_{2}, \ell_{3}$, at angles $0, \pi / 3$, and $2 \pi / 3$.

Let $X, Y, Z, X^{\prime}, Y^{\prime}, Z^{\prime}$ denote the six wedges in counterclockwise order, labeled as in Fig. 3. Note that the angle between the $x$-axis and any edge $p_{i-1} p_{i}$ of a tour with $p_{i-1} \in X$ and $p_{i} \in X^{\prime}$, say, belongs to the interval $[0, \pi / 3]$. A piece $p_{i-1} p_{i} p_{i+1}$ of a tour is of the form $X X^{\prime} X$, say, if $p_{i-1}, p_{i+1} \in X$ and $p_{i} \in X^{\prime}$.

Observation 1. Consider a piece of a tour, which is of the form $X Q X$, where $Q=Y^{\prime}, X^{\prime}$, or $Z$. Then the rotation angle at the middle point of this piece, which belongs to $Q$, is at most $2 \pi / 3$. The same holds for any other piece consisting of two edges, which starts and ends in the same wedge, and whose middle point belongs to one of the three opposite wedges.

Observation 2. Consider a piece of a tour, which is of the form $X X^{\prime} Y$ or $X X^{\prime} Z^{\prime}$. Then the rotation angle at the middle point of this piece, which belongs to $X^{\prime}$, is at most $2 \pi / 3$. The same holds for any other piece of the form $X^{\prime} X Z, X^{\prime} X Y^{\prime}, Y Y^{\prime} X, Y Y^{\prime} Z, Y^{\prime} Y X^{\prime}$, $Y^{\prime} Y Z^{\prime}, Z Z^{\prime} Y, Z Z^{\prime} X^{\prime}, Z^{\prime} Z X, Z^{\prime} Z Y^{\prime}$.

Proof of Theorem 1. We distinguish two cases:

Case 1. There are at most two nonempty double wedges. If all points are contained in a unique double wedge, say $X X^{\prime}$, then by Observation 1, they can be connected by an acute tour of the form $\left(X X^{\prime}\right)^{*}$. The tours starts in $X$, ends in $X^{\prime}$, and alternates between the wedges $X$ and $X^{\prime}$ until all points in $X \cup X^{\prime}$ are exhausted. Assume now that there are exactly two nonempty double wedges, $X X^{\prime}$ and $Y Y^{\prime}$, say, and refer to Fig. 4. Consider a spanning tour of the form $\left(X X^{\prime}\right)^{*}\left(Y Y^{\prime}\right)^{*}$, where $\left(X X^{\prime}\right)^{*}$ and $\left(Y Y^{\prime}\right)^{*}$ are point sequences that alternate between the corresponding opposite wedges until all points in those wedges are exhausted. By Observations 1 and 2, at each vertex of this tour the rotation angle is at most $2 \pi / 3$. 


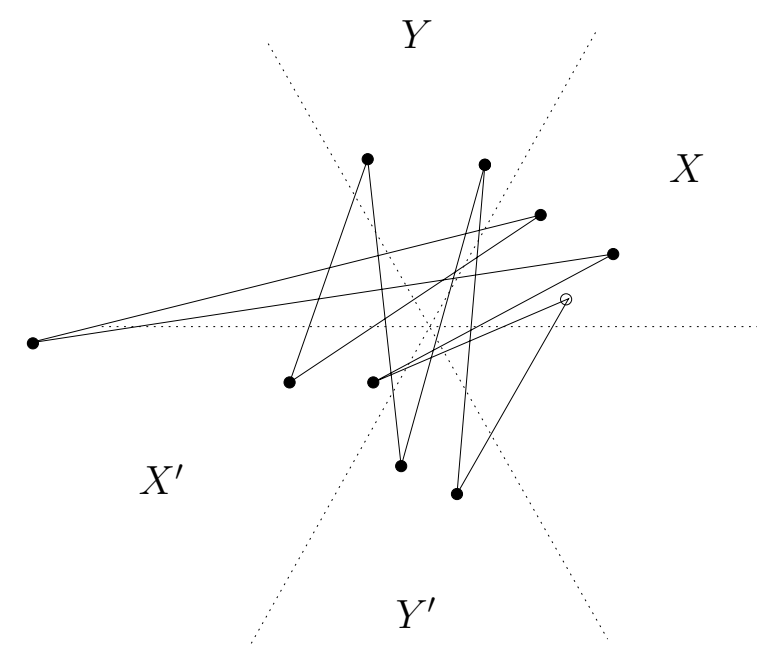

Figure 4: Case 1: points in two double wedges. A tour of the form $X X^{\prime} X X^{\prime} X X^{\prime} Y Y^{\prime} Y Y^{\prime}$ is shown; its starting vertex in $X$ is drawn as an empty circle.
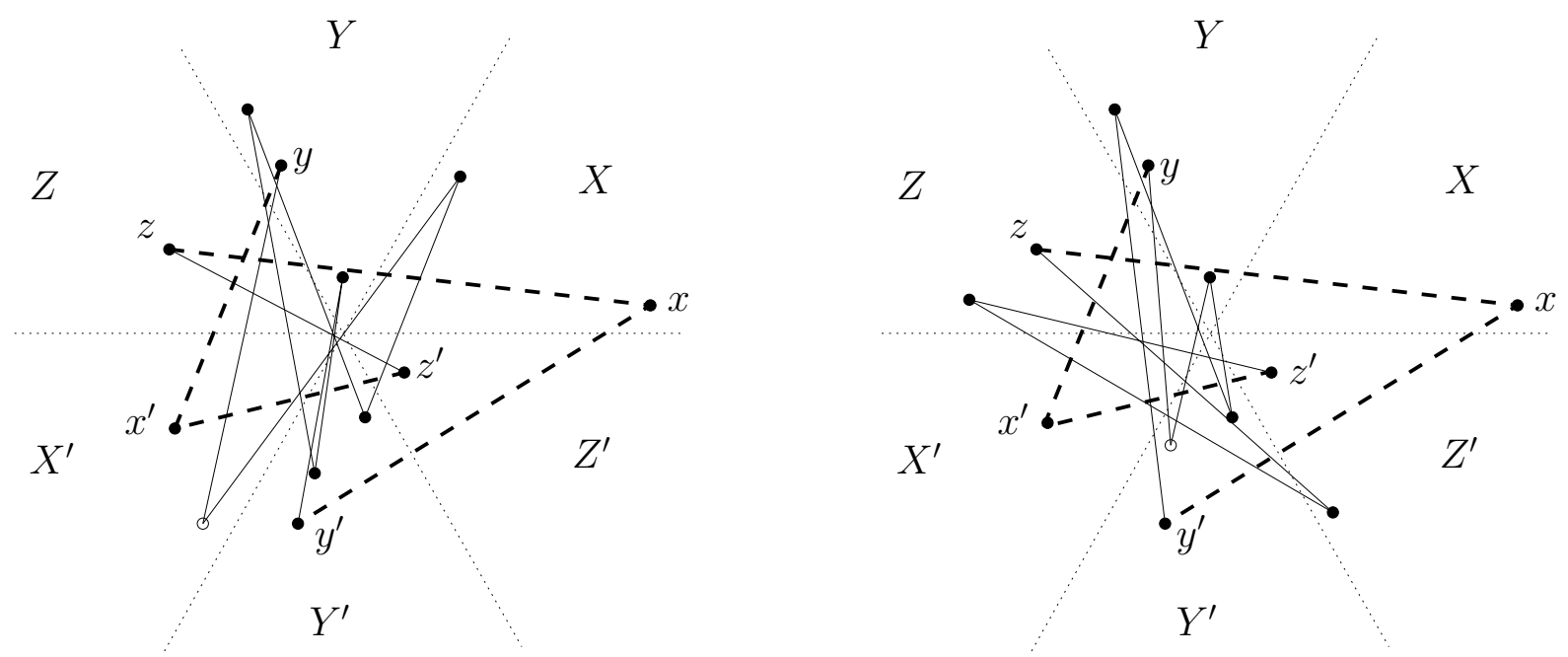

Figure 5: Case 2: points in three double wedges. Left: a tour of the form $X^{\prime} X Y^{\prime} Y Y^{\prime} Y y^{\prime} x z z^{\prime} x^{\prime} y$ is shown; its starting vertex in $X^{\prime}$ is drawn as an empty circle. Right: a tour of the form $Y^{\prime} Y Y^{\prime} Y y^{\prime} x z Z^{\prime} Z z^{\prime} x^{\prime} y$ is shown; its starting vertex in $Y^{\prime}$ is drawn as an empty circle. 
Case 2. There are exactly three nonempty double wedges; refer to Fig. 5. Arbitrarily pick one point from each wedge: $x \in X, y \in Y, z \in Z, x^{\prime} \in X^{\prime}, y^{\prime} \in Y^{\prime}, z^{\prime} \in Z^{\prime}$. Consider the two triangles $\Delta x z y^{\prime}$ and $\Delta y x^{\prime} z^{\prime}$. The sum of the interior angles of the two triangles is obviously $2 \pi$. By averaging, there is one pair of points lying in opposite wedges, say $x$ and $x^{\prime}$, whose angles sum up to at most $2 \pi / 3$. Thus, each of these angles is at most $2 \pi / 3$ : $\angle z x y^{\prime} \leqslant 2 \pi / 3$, and $\angle y x^{\prime} z^{\prime} \leqslant 2 \pi / 3$.

If $|X \cap S|=\left|X^{\prime} \cap S\right| \geqslant 2$, consider a spanning tour $\left(X^{\prime} X\right)^{+}\left(Y^{\prime} Y\right)^{+} y^{\prime} x z\left(Z^{\prime} Z\right)^{+} z^{\prime} x^{\prime} y$. Here $\left(X^{\prime} X\right)^{+}$denotes a nonempty alternating path between the wedges $X^{\prime}$ and $X$, that starts in $X^{\prime}$, ends in $X$, and involves all points except $x$ and $x^{\prime}$. The notations $\left(Y^{\prime} Y\right)^{+}$and $\left(Z^{\prime} Z\right)^{+}$are used analogously. An example is depicted in Fig. 5 (left). By Observations 1 and 2 , and by our choice of $x, y, z, x^{\prime}, y^{\prime}, z^{\prime}$, all rotation angles along this tour are at most $2 \pi / 3$, as required.

If $|X \cap S|=\left|X^{\prime} \cap S\right|=1$, consider a spanning tour $\left(Y^{\prime} Y\right)^{+} y^{\prime} x z\left(Z^{\prime} Z\right)^{+} z^{\prime} x^{\prime} y$; see Fig. 5 (right). The arguments justifying that all rotation angles are at most $2 \pi / 3$ are the same as before.

The proof of Theorem 1 is now complete.

\section{Covering by two acute tours}

Proof of Theorem 2. (i) Take a horizontal line $\ell$ and a partition of our point set $S=S^{+} \cup S^{-}$into two subsets, each of size $n / 2$, such that $S^{+}$and $S^{-}$are in the closed half-planes above and below $\ell$, respectively. If some points of $S$ lie on $\ell$, we can include them in either of these sets so as to satisfy the condition. Next, take a vertical line $\ell^{\prime}$ which gives rise to another equipartition of $S$. Assume for simplicity that $\ell$ and $\ell^{\prime}$ coincide with the $x$ and $y$ coordinate axes. See Fig. 6, for an illustration.

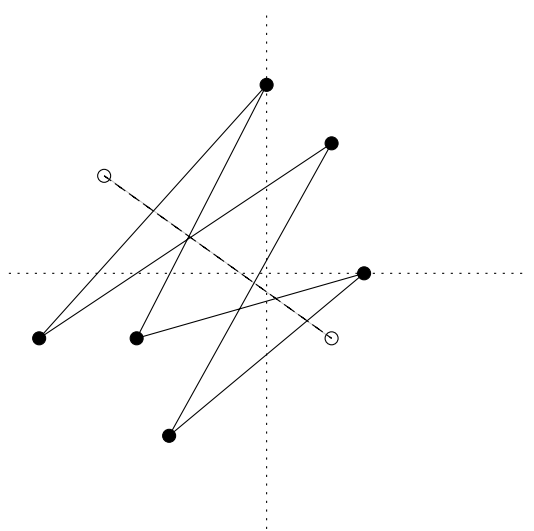

Figure 6: Even set covered by two tours with 6 and 2 points, respectively; $a=3$, and $b=1$. (A double-edge counts as a tour.)

Thus, we obtain a partition $S=S_{1} \cup S_{2} \cup S_{3} \cup S_{4}$ such that all points of $S_{i}$ belong to the $i$ th closed quadrant determined by the axes (enumerated in the counterclockwise 
order), $\left|S_{1}\right|=\left|S_{3}\right|=a$, and $\left|S_{2}\right|=\left|S_{4}\right|=b$ for some integers $a$ and $b$ with $a+b=n / 2$. Connect now all elements of $S_{1} \cup S_{3}$ by a tour of length $2 a$ alternating between $S_{1}$ and $S_{3}$. Similarly, connect the elements of $S_{2} \cup S_{4}$ by an alternating tour of length $2 b$. Obviously, both tours are acute. The above procedure can be performed in linear time, using any linear time selection algorithm [9].

(ii) Find two orthogonal lines and a partition $S=S_{1} \cup S_{2} \cup S_{3} \cup S_{4}$ satisfying the conditions of Lemma 1. Using the notation of the proof of part (i), now we have $a=\left\lfloor\frac{n}{4}\right\rfloor$ and $b=\left\lceil\frac{n}{4}\right\rceil$. As above, we obtain two acute tours, of lengths $2\left\lfloor\frac{n}{4}\right\rfloor$ and $2\left\lceil\frac{n}{4}\right\rceil$, respectively. This completes the proof of part (ii) of Theorem 2.

By keeping only the larger tour, Theorem 2 immediately implies

Corollary 1. For any even n, every n-element point set in the plane admits an acute even tour covering at least half of its elements.

\section{Acute tours for points in convex position}

Throughout this section, let $S$ denote a set of $n \geqslant 8$ points in the plane, in convex position and let $S=S_{1} \cup S_{2} \cup S_{3} \cup S_{4}$ be a partition satisfying the conditions in Lemma 1. A 3 -edge path (on 4 points) is called a hook if the rotation angles at its two intermediate vertices are acute.

Lemma 3. Let $P=\left\{p_{1}, p_{2}, p_{3}, p_{4}\right\}$ be the vertex set of a convex quadrilateral, with $p_{i} \in S_{i}$, $i=1,2,3,4$. Then at least one of the following two conditions is satisfied.

(i) $p_{1} p_{3} p_{4} p_{2}$ and $p_{3} p_{1} p_{2} p_{4}$ are hooks, or

(ii) $p_{1} p_{3} p_{2} p_{4}$ and $p_{3} p_{1} p_{4} p_{2}$ are hooks.

Proof. At least one of the two angles defined by the diagonals $p_{1} p_{3}$ and $p_{2} p_{4}$ is larger or equal to $\pi / 2$. Let $x$ denote the crossing point of these diagonals. If $\angle p_{1} x p_{2} \geqslant \pi / 2$, then the two 3 -edge paths $p_{1} p_{3} p_{4} p_{2}$ and $p_{3} p_{1} p_{2} p_{4}$ are hooks, while if $\angle p_{2} x p_{3} \geqslant \pi / 2$, then $p_{1} p_{3} p_{2} p_{4}$ and $p_{3} p_{1} p_{4} p_{2}$ are hooks.

We say that a convex quadrilateral $P$, as in Lemma 3 , is of type 1 if $\angle p_{1} x p_{2} \geqslant \pi / 2$, and of type 2 , otherwise (i.e., if $\angle p_{2} x p_{3}>\pi / 2$ ).

Lemma 4. Let $P=\left\{p_{1}, p_{2}, p_{3}, p_{4}\right\}, Q=\left\{q_{1}, q_{2}, q_{3}, q_{4}\right\}$, and $R=\left\{r_{1}, r_{2}, r_{3}, r_{4}\right\}$ be three vertex-disjoint convex quadrilaterals with $p_{i}, q_{i}, r_{i} \in S_{i}$, for $i=1,2,3,4$. Then there exist two hooks induced by two of these quadrilaterals such that the two endpoints of the first one and the two endpoints of the second one lie in different parts of the partition $S_{1} \cup S_{2} \cup S_{3} \cup S_{4}$. Two such hooks are called opposite. (See Fig. 7 (left).)

Proof. By the pigeonhole principle, two out of the three quadrilaterals, say $P$ and $Q$, must have the same type. By Lemma 3, one can find a hook in each of them such that their endpoints are all in different parts of the partition, i.e., two opposite hooks. 

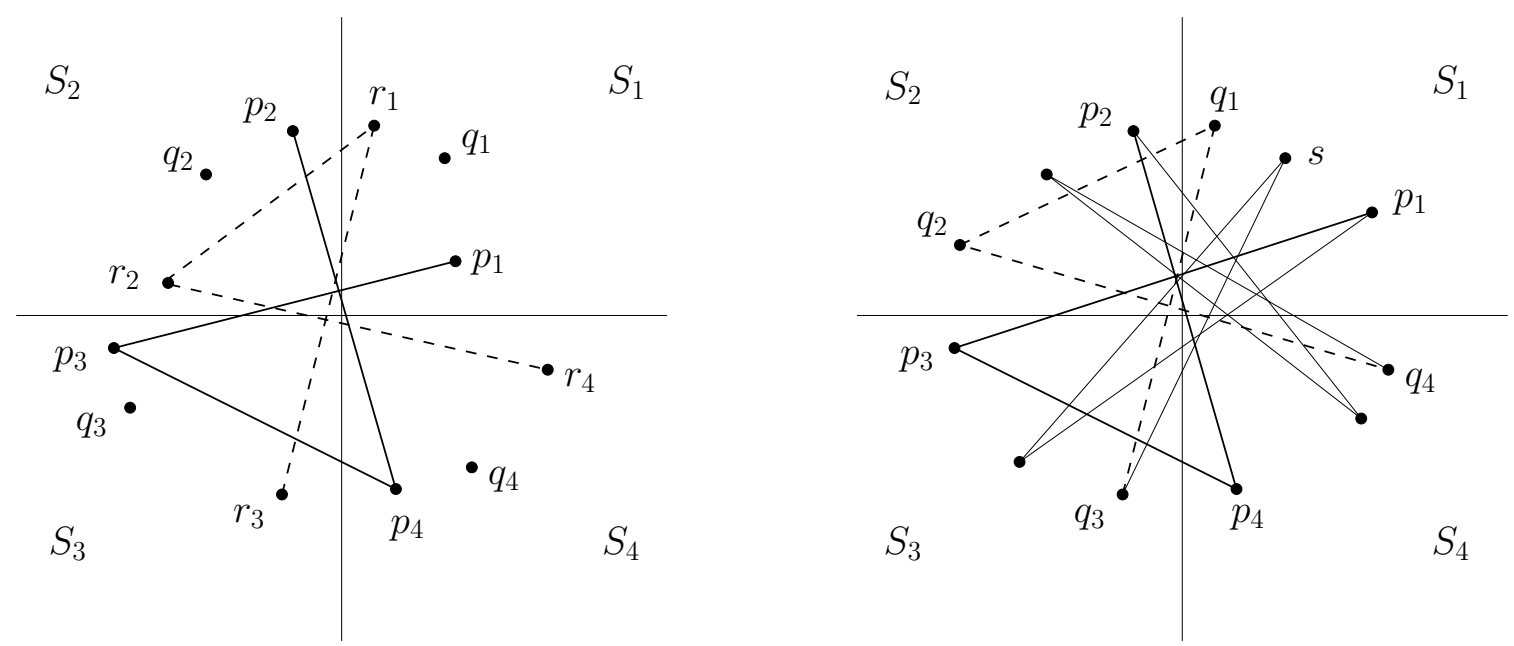

Figure 7: Left: $p_{1} p_{3} p_{4} p_{2}$ and $r_{3} r_{1} r_{2} r_{4}$ are two opposite hooks. Right: an acute tour of $S$ of the form $\left(S_{1} S_{3}\right)^{+} p_{1} p_{3} p_{4} p_{2}\left(S_{4} S_{2}\right)^{+} q_{4} q_{2} q_{1} q_{3}$, starting at $s \in S_{1}$.

Proof of Theorem 3. Consider a partition $S=S_{1} \cup S_{2} \cup S_{3} \cup S_{4}$ satisfying the conditions in Lemma 1. Since $|S| \geqslant 12$, we have $\left|S_{i}\right| \geqslant 3$. Pick 3 points from each $S_{i}$, and using these points construct three vertex-disjoint convex quadrilaterals, $P, Q$, and $R$. By Lemma 4, two of these quadrilaterals, $P$ and $Q$, say, determine opposite vertex-disjoint hooks. Suppose without loss of generality that $P$ and $Q$ are of type 1 , and these two hooks are $p_{1} p_{3} p_{4} p_{2}$ and $q_{4} q_{2} q_{1} q_{3}$, where $p_{i}, q_{i} \in S_{i}, i=1,2,3,4$. See Fig. 7(right).

Let $\left(S_{i} S_{j}\right)^{+}$denote a polygonal path starting in $S_{i}$, ending in $S_{j}$, alternating between $S_{i}$ and $S_{j}$, and exhausting all points of $S_{i} \cup S_{j}$, except for $p_{i}, p_{j}, q_{i}, q_{j}$. The following tour is acute: $\left(S_{1} S_{3}\right)^{+} p_{1} p_{3} p_{4} p_{2}\left(S_{4} S_{2}\right)^{+} q_{4} q_{2} q_{1} q_{3}$, and this completes the proof.

\section{Random point sets}

We first verify Theorem 4 for centrally symmetric convex bodies, and then in its full generality.

Lemma 5. Let $B$ be a centrally symmetric convex body in the plane and let $S$ be a set of $n$ points, randomly and uniformly selected from $B$. Then, for any $\varepsilon>0, S$ almost surely admits a spanning tour with no rotation angle larger than $\varepsilon$, as $n$ tends to infinity.

Proof. Let $\varepsilon$ be fixed, and let $o$ denote the center of $B$. Assume without loss of generality that area $(B)=1$. Any chord through $o$ divides the area of $B$ into two equal parts. Therefore, there is a positive constant $\delta=\delta(B, \varepsilon)$, depending only on $B$ and $\varepsilon$, such that for every wedge $W$ with angle at most $\pi-\frac{\varepsilon}{2}$ and apex at $o$, we have that $\operatorname{area}(W \cap B) \leqslant 1 / 2-\delta$. Let $m=\lceil n / 2\rceil$.

Let $p_{1}, p_{2}, \ldots p_{n}$ be $n$ random points, independently and uniformly selected from $B$, listed in their circular order of visibility from $o$. The indices are taken modulo $n$, so that $p_{n+1}=p_{1}$. Note that almost surely all points $p_{i}$ are distinct and different from $o$. 
If $n$ is odd, consider the spanning tour $C=p_{1} p_{m+1} p_{2} p_{m+2} \ldots p_{m} p_{1}$. For every $i$, almost surely we have

$$
\pi-\frac{\varepsilon}{2} \leqslant \angle p_{i} o p_{m+i-1} \leqslant \pi+\frac{\varepsilon}{2},
$$

and

$$
\pi-\frac{\varepsilon}{2} \leqslant \angle p_{i} o p_{m+i} \leqslant \pi+\frac{\varepsilon}{2} .
$$

Therefore, we almost surely have $\angle p_{m+i-1} p_{i} p_{m+i} \leqslant \varepsilon$, for every $i$, and the tour $C$ meets the requirements.

If $n$ is even, we choose two odd numbers $n_{1}, n_{2}$ with $n_{1}+n_{2}=n$ such that $0 \leqslant$ $n_{1}-n_{2} \leqslant 2$. That is, $n_{1}$ is $m$ or $m+1$ while $n_{2}$ is $m$ or $m-1$. Connect the points $p_{i}$ by two disjoint cycles, $C_{1}$ and $C_{2}$, of length $n_{1}$ and $n_{2}$, with property that (1) in the cyclic order around $o$, the points $p_{1}, p_{2}, \ldots$ belong alternately to $C_{1}$ and $C_{2}$, as much as possible; and (2) every edge of $C_{1}$ and $C_{2}$ connects two points, $p_{i}$ and $p_{j}$, with $|j-i-m| \leqslant 3(\bmod$ $n)$. We distinguish two cases.

Case 1. $n_{1}=n_{2}=m$. Let

$$
\begin{aligned}
& C_{1}=p_{1} p_{2+m} p_{3} p_{4+m} p_{5} \ldots p_{n-1} p_{m}, \\
& C_{2}=p_{2} p_{3+m} p_{4} p_{5+m} p_{6} \ldots p_{n} p_{1+m} .
\end{aligned}
$$

Switching between these two cycles at two points, we can combine them into a single spanning tour $C$, as follows.

$$
C=p_{1} p_{2+m} p_{3} p_{4+m} p_{5} \ldots p_{n-1} p_{m} p_{2} p_{3+m} p_{4} p_{5+m} p_{6} \ldots p_{n} p_{1+m} .
$$

It remains true that $|j-i-m| \leqslant 3(\bmod n)$ for every edge $p_{i} p_{j}$ of $C$, so that almost surely all rotation angles of $C$ will be smaller than $\varepsilon$.

Case 2. $n_{1}=m+1, n_{2}=m-1$. Let

$$
\begin{aligned}
& C_{1}=p_{1} p_{2+m} p_{3} p_{4+m} p_{5} \ldots p_{n} p_{m+1}, \\
& C_{2}=p_{2} p_{3+m} p_{4} p_{5+m} p_{6} \ldots p_{n-1} p_{m} .
\end{aligned}
$$

We can combine them into a single spanning tour $C$, as follows.

$$
C=p_{1} p_{2+m} p_{3} p_{4+m} p_{5} \ldots p_{n} p_{m+1} p_{2} p_{3+m} p_{4} p_{5+m} p_{6} \ldots p_{n-1} p_{m}
$$

It remains true that $|j-i-m| \leqslant 3(\bmod n)$ for every edge $p_{i} p_{j}$ of $C$, so that almost surely all rotation angles of $C$ will be smaller than $\varepsilon$.

To prove Theorem 4 in its full generality, we need the following technical lemma. Its proof is very similar to that of Lemma 5. The minor modifications are left to the reader.

Lemma 6. Let $B$ be a centrally symmetric convex set in the plane with nonempty interior. Let $o$ denote the center of $B$, let $\varepsilon>0$ be fixed, let $s$ and $t$ be two points of $B$, and let $S^{\prime}$ be a set of at most $\varepsilon n / 4$ points not belonging to $B$. 
Then, for any set $S$ of $n$ points randomly and uniformly selected from $B$, the set $S \cup S^{\prime}$ almost surely admits a spanning path satisfying the following conditions, as $n \rightarrow \infty$ :

(i) all of its turning angles are at most $\varepsilon$;

(ii) its first two points are $p_{1}$ and $p_{2}$ such that $\angle o p_{1} p_{2} \leqslant \varepsilon / 3$, and $\angle s o p_{1} \leqslant \varepsilon / 3$;

(iii) its last two points are $q_{2}$ and $q_{1}$ such that $\angle o q_{1} q_{2} \leqslant \varepsilon / 3$, and $\angle t o q_{1} \leqslant \varepsilon / 3$.

Proof of Theorem 4. Assume without loss of generality that $\operatorname{area}(B)=1$. Consider a square lattice of minimum distance $\delta$, for some $\delta>0$ to be specified later. Let $A=A(\delta)$ denote the total area of all cells (lattice squares of side length $\delta$ ) completely contained in $B$, and let $A^{\prime}=A^{\prime}(\delta)$ denote the total area of all those cells that intersect $B$, but are not completely contained in it. Obviously, $A+A^{\prime} \geqslant 1$. Since the boundary of $B$ is the union of finitely many rectifiable curves, we have

$$
\lim _{\delta \rightarrow 0} A=1, \quad \limsup _{\delta \rightarrow 0} \frac{A^{\prime}}{\delta}<\infty .
$$

Therefore, we can choose $\delta>0$ so that $A^{\prime} \leqslant \varepsilon / 6$.

Let $X_{1}, X_{2}, \ldots, X_{m}$ denote the cells completely contained in $B$, in some arbitrary order, and let $o_{i}$ denote the center of $X_{i}$. For any $1 \leqslant i \leqslant m$, let $s_{i}$ be a point on the line $o_{i} O_{i-1}$ such that $o_{i}$ belongs to the segment $s_{i} o_{i-1}$. Analogously, let $t_{i}$ be a point on the line $o_{i} O_{i+1}$ such that $o_{i}$ belongs to the segment $t_{i} o_{i+1}$. Here the indices are taken modulo $m$.

Let $S$ be a set of $n$ points in $B$, selected independently, randomly, and uniformly. Let $S_{i}=S \cap X_{i}$, for $1 \leqslant i \leqslant m$, and let $S^{\prime}=S \backslash \cup_{i=1}^{m} S_{i}$. Divide $S^{\prime}$ into $m$ almost equal parts, $S_{1}^{\prime}, S_{2}^{\prime}, \ldots, S_{m}^{\prime}$ with $\left\|S_{i}^{\prime}|-| S_{j}^{\prime}\right\| \leqslant 1$, for any $i, j=1, \ldots, m$.

For each $1 \leqslant i \leqslant m$, apply Lemma 6 with $S_{i}, S_{i}^{\prime}, s_{i}$, and $t_{i}$, to obtain a spanning path $P_{i}$. The spanning tour $P_{1} P_{2} \ldots P_{m}$ obtained by the concatenation of these paths now meets the requirements.

\section{References}

[1] E. Ackerman, O. Aichholzer, and B. Keszegh: Improved upper bounds on the reflexivity of point sets, Computational Geometry: Theory and Applications, 42(3) (2009), 241-249.

[2] A. Aggarwal, D. Coppersmith, S. Khanna, R. Motwani, and B. Schieber: The angular-metric traveling salesman problem, SIAM Journal on Computing, 29(3) (1999), 697-711.

[3] O. Aichholzer, T. Hackl, M. Hoffmann, C. Huemer, A. Pór, F. Santos, B. Speckman, and B. Vogtenhuber: Maximizing maximal angles for plane straight-line graphs, Proceedings of the 10th International Workshop on Algorithms and Data Structures, 2007, vol. 4619/2007 of LNCS, pp. 458-469.

[4] E. M. Arkin, M. A. Bender, E. D. Demaine, S. P. Fekete, J. S. B. Mitchell, and S. Sethia: Optimal covering tours with turn costs, SIAM Journal on Computing, 35(3) (2005), 531-566. 
[5] E. M. Arkin, S. Fekete, F. Hurtado, J. Mitchell, M. Noy, V. Sacristán, and S. Sethia: On the reflexivity of point sets, in Discrete and Computational Geometry: The Goodman-Pollack Festschrift (B. Aronov, S. Basu, J. Pach, M. Sharir, editors), Springer, 2003, pp. 139-156.

[6] I. Bárány, A. Pór, and P. Valtr: Paths with no small angles, Proceedings of the LATIN Theoretical Informatics Conference, 2008, vol. 4957/2008 of LNCS, pp. 654-663.

[7] J. Boissonat, A. Cérézo, and J. Leblond: Shortest paths of bounded curvature in the plane, Journal of Intelligent and Robotic Systems: Theory and Applications, 11 (1994), 5-20.

[8] T. Chan: Remarks on $k$-level algorithms in the plane, manuscript, Univ. of Waterloo, 1999.

[9] T. Cormen, C. Leiserson, R. Rivest and C. Stein: Introduction to Algorithms, second edition, McGraw-Hill, 2001.

[10] R. Courant and H. Robbins: What is Mathematics? An Elementary Approach to Ideas and Methods, Oxford University Press, New York, 1979.

[11] T. K. Dey: Improved bounds on planar $k$-sets and related problems, Discrete $\&$ Computational Geometry, 19 (1998), 373-382.

[12] S. P. Fekete and G. J. Woeginger: Angle-restricted tours in the plane, Computational Geometry: Theory and Applications, 8(4) (1997), 195-218.

[13] T. Frachard: Smooth trajectory planning for a car in a structured world, Proceedings of the IEEE International Conference on Robotics, 1989, pp. 318-323.

[14] P. Jacobs and J. Canny: Planning smooth paths for mobile robots, Proceedings of the IEEE International Conference on Robotics, 1989, pp. 2-7.

[15] L. Lovász: On the number of halving lines, Ann. Univ. Sci. Budapest, Eötvös, Sec. Math., 14 (1971), 107-108. 